\title{
Relación entre el Patrón Dactiloscópico Epidérmico y Dérmico
}

\author{
Relationship Between the Epidermal and the Dermal Dactyloscopic Pattern
}

\author{
Juan Silva ${ }^{1,2}$; Claudia Araya ${ }^{1}$; Miguel Soto²; Sebastián González²; Andrés Salcedo ${ }^{3}$; Patricio Bustos ${ }^{4}$; \\ Ismael Ilufi ${ }^{4}$; Jose Ormazábal ${ }^{4}$; Juan Sanhueza ${ }^{4}$; Alfonso Pino ${ }^{4}$; Alex Plaza ${ }^{4}$ \& Sebastián Arcos ${ }^{1}$
}

SILVA, J. ; ARAYA, C.; SOTO, M.; GONZÁleZ, S.; SALCEDO, A.; BUSTOS, P.; ILUFI, I.; ORMAZABAL, J.; SANHUEZA, J.; PINO, A.; PLAZA, A. \& ARCOS, S. Relación entre el patrón dactiloscópico epidérmico y dérmico. Int. J. Morphol., 36(4):1290-1297, 2018.

RESUMEN: El método más utilizado en identificación humana es el dactiloscópico, que registra, analiza y coteja los tipos fundamentales y puntos característicos de las figuras presentes en el dactilograma, determinando el grado de coincidencia, entre un patrón de identidad dubitada y uno de identidad indubitada. Debido a los procesos que afectan la piel de cadáveres, como la putrefacción entre otros, se utilizan las técnicas necropapiloscópicas que ocupan los patrones dérmicos para la identificación humana. El objetivo del presente trabajo es comparar registros epidérmicos con dérmicos, y validar científicamente, este método de identificación (epidérmico - dérmico). Para ello se utilizaron 32 dedos de cadáveres de individuos chilenos, de ambos sexos y de entre 40 y 80 años. Para obtener el registro físico artificial epidérmico y dérmico se utilizó la técnica de obtención de impresiones necrodactilares y se comparó a través de la técnica de cotejo dactiloscópico. Estos procedimientos fueron realizados por peritos criminalísticos de Carabineros de Chile. Se logró evidenciar diferencias importantes entre epidermis y dermis en cuanto a cantidad y calidad de puntos característicos y presencia de líneas seniles. En relación a tipos fundamentales, se evidenció que en dermis es difícil la observación de tipos fundamentales (borrosos) pero no se encontró ningún dígito que presentara tipo fundamental diferente entre epidermis y dermis. También se constataron diferencias por sexo y edad. Finalmente se pudo evidenciar, que existe una relación morfológica semejante entre registros físicos artificiales de patrones papiloscópicos epidérmicos con dérmicos provenientes del mismo individuo. Esto permite utilizar los registros dérmicos para la identificación humana positiva. Los resultados de este trabajo son importantes al aportar evidencia científica para la identificación humana en base al patrón dactiloscópico dérmico.

PALABRAS CLAVE: Necropapiloscopía; Dactilograma; Dactiloscopía; Papiloscopía; Identificación humana.

\section{INTRODUCCIÓN}

Durante la historia de la humanidad han sido utilizados un gran número de métodos de identificación humana; el método empírico, el nominativo, el antropométrico, el otométrico, el craneométrico, el oftálmico, el dentario, el rugoscópico, el queiloscópico, el ungueal, el seroinmunológico, el genético y el dactiloscópico o papiloscópico. Este último método ha sido el más utilizado (Teke Schlicht, 2010), debido a las características propias del dactilograma (únicos, particulares, inmutables, perennes, fáciles de observar, fáciles de comparar y de almacenar) (Galton, 1892; Rico Méndez \& de Anda, 1991; Kücken \& Newell, 2004).

El patrón dactiloscópico, papiloscópico o dactilograma, corresponde al conjunto de crestas, poros sudoríparos y surcos característicos de la superficie de la piel de la cara volar de dedos, palmas de manos y plantas de pies y se caracteriza por poseer un ordenamiento que conforma configuraciones únicas y fácilmente distinguibles (Gibbs, 1967; de Antón \& Barberá, 2005).

La dactiloscopía o papiloscopía corresponde al estudio científico de las impresiones digitales, palmares y plantares, que tiene por finalidad la identificación infalible o indubitada del individuo (Reyna, 1909; Trujillo Arriaga, 2007).

Esta disciplina, utiliza estos patrones para determinar la identidad de los individuos, debido a sus características particulares, aprovechando sus patrones morfológicos denominados grupos o tipos fundamentales (TF), tales como el arco, la presilla interna, la presilla externa y el verticilo

\footnotetext{
${ }^{1}$ Facultad de Medicina, Universidad San Sebastian, Santiago, Chile.

${ }^{2}$ Departamento de Anatomía y Medicina Legal, Facultad de Medicina, Universidad de Chile, Santiago, Chile.

${ }^{3}$ Facultad de Odontología, Universidad San Sebastian, Santiago, Chile.

${ }^{4}$ Laboratorio de Criminalística de Carabineros de Chile (LABOCAR). Chile.
} 
(Holt, 1961; de Wilde et al., 1981; Kücken \& Newell, 2005; Kücken, 2007) y los puntos característicos (PC), que son las formas especiales que pueden observarse en las líneas del dactilograma (encierro, gancho, bifurcación, empalme, enlazada, punto, final de línea, interrupción, línea desviada, transversal, fragmento de línea, excrecencias) (Ilabaca et al., 1986). En la práctica existe semejanza entre algunos de estos PC, lo que ha determinado que los más útiles y utilizados en la identificación humana sean la bifurcación, el encierro, el gancho, el islote, el punto, la línea interrumpida y la línea libre (González Wood, 1946). En Chile se utiliza una modificación de los PC descritos por este último autor (extremo de línea, bifurcación-horquilla, gancho, encierro, empalme, islote y punto). También es importante destacar que por dedo se puede llegar a observar entre 45 a 80 PC, siendo estos hitos morfológicos particulares por su forma, por su ubicación y por su dirección, los que constituyen un factor de individualidad personal (Vucetich, 1904; Aliaga, 1988) (Fig. 1).

La identificación humana se lleva a cabo a través de un proceso de comparación en el que se cotejan características de un individuo cuya identidad es desconocida o dubitada con características de individuos conocidos o de identidad indubitada (que se encuentran filiados o registrados legalmente). Así en la identificación dactiloscópica o papiloscópica, el análisis de los TF y de los PC, permite determinar la coincidencia entre una huella dactilar (de identidad dubitada) y una impresión digital (de identidad indubitada). En este procedimiento se admite como norma internacional, que cuando se encuentran de nueve a doce puntos característicos exactamente iguales (en forma, situación, distancia y relación) y ninguno discordante, se puede afirmar la identificación positiva del individuo (Beaven, 2003; Lubian, 2006).
Lamentablemente, no siempre es posible registrar en forma adecuada, el patrón dactiloscópico o papiloscópico epidérmico externo o superficial (también denominado relieve papilar epidérmico externo) de un cadáver de individuo de identidad desconocida. En estos casos será necesario utilizar las técnicas propias de la necropapiloscopía; disciplina derivada de la dactiloscopia o papiloscopía y que está orientada a la identificación de cadáveres de identidad dubitada y cuyo estado al instante de su hallazgo presenta dificultad para la obtención de documentaciones papiloscopicas de valor identificatorio (Girotti, 2014).

Estas técnicas son utilizadas, sobre todo en aquellos cadáveres donde el proceso de putrefacción o descomposición está avanzado, impidiendo observar los detalles propios del patrón dactiloscópico epidérmico superficial, debido a la completa o parcial destrucción de la epidermis. En estos casos la capa más profunda (dermis), puede revelar detalles discernibles del patrón dactiloscópico dérmico o relieve papilar dérmico (Holder et al., 2012).

El valor de este método de cotejo, estaría basado en que las proyecciones epidérmicas profundas determinan impresiones en negativo en la dermis, que presentarían una relación semejante, pero no igual, entre epidermis y dermis (Chacko \& Vaidya, 1968).

En este método de identificación de cadáveres, hemos constatado un problema complejo, debido a que la comparación se realiza entre un patrón papiloscópico dérmico (del cual no existe registro) proveniente del cadáver de identidad desconocida con un patrón papiloscópico epidérmico superficial (proveniente de la filiación legal y obtenido de individuos vivos) (Fig. 2). Este método de identificación (epidérmico - dérmico) asume que la morfología del patrón

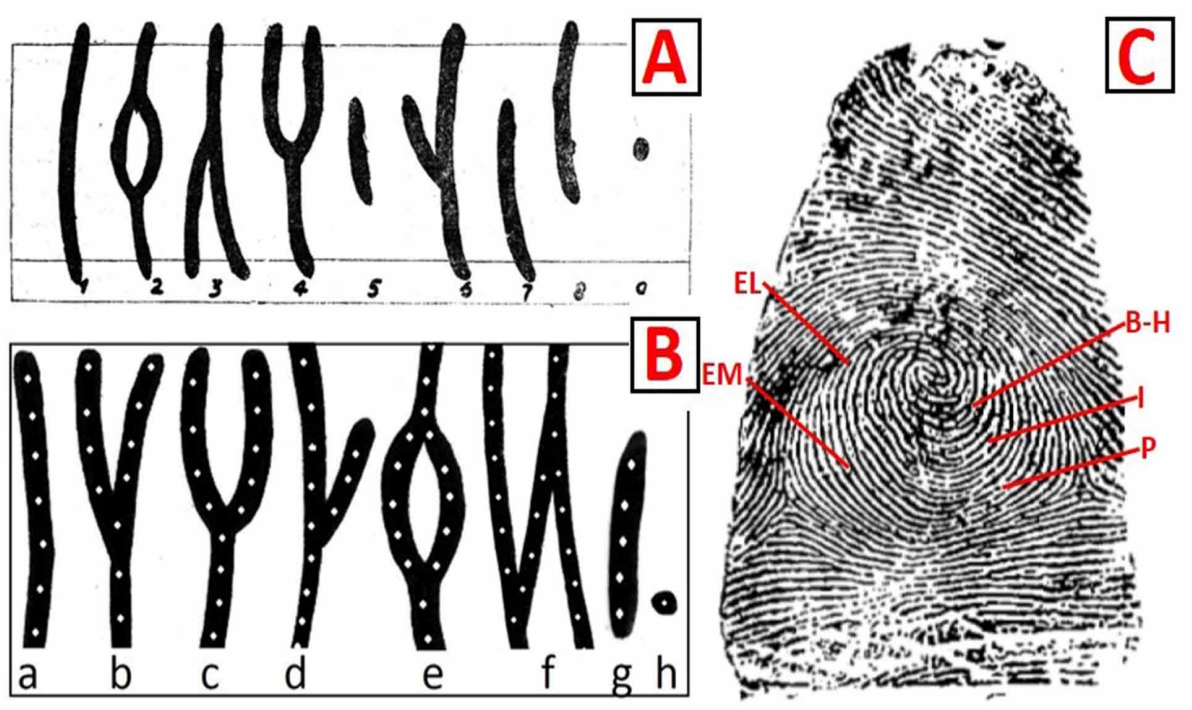

Fig. 1. En A, PC descritos por González Wood (1946); 1- línea libre. 2- encierro. 3- horquilla. 4- bifurcación. 5- islote. 6- gancho. 7comienzo de línea. 8- final de línea. 9- punto. En B, PC utilizados en Chile (tomados y modificados de González Wood, 1946); a- extremo de línea. b- horquilla. c- bifurcación. d- gancho. e- encierro. f- empalme. g- islote. h- punto. En C, algunos ejemplos de PC observados en un registro físico artificial epidérmico superficial; EL- extremo de línea. EM- empalme. B-H- bifurcación u horquilla. I- islote. P- punto. 

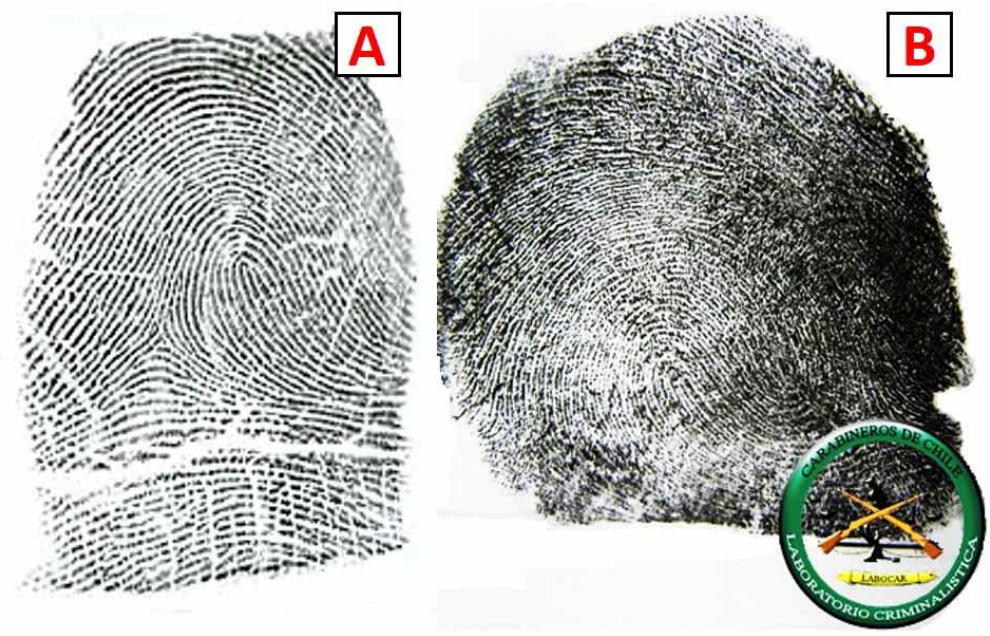

Fig. 2. En A, ejemplo de registro físico artificial de patrón papiloscópico epidérmico.

En B, ejemplo de registro físico artificial de patrón papiloscópico dérmico. Obsérvese que en el primero, las líneas se ven simples y continuas, mientras que en el segundo las líneas se observan dobles y discontinuas (asemejando un collar de cuentas).

papiloscópico epidérmico superficial, es semejante o igual, a la morfología del patrón papiloscópico dérmico, sin considerar que se están comparando tejidos orgánicos distintos y que esto podría conllevar diferencias. No hemos encontrado en la literatura especializada, estudios que corroboren científicamente este método de identificación (epidérmico - dérmico).

El objetivo del presente trabajo es comparar registros físicos artificiales de patrones papiloscópicos epidérmicos con registros físicos artificiales de patrones papiloscópicos dérmicos de individuos adultos chilenos, así podremos determinar las semejanzas, y posibles diferencias, entre estos patrones, con el fin último de dar respaldo científico a este método de identificación (epidérmico - dérmico).

\section{MATERIAL Y MÉTODO}

Para este estudio se utilizaron 32 dedos provenientes de cadáveres congelados de individuos chilenos, de ambos sexos y con edades que fluctuaron entre los 40 y 80 años, pertenecientes a la Unidad de Anatomía y Medicina Legal de la Facultad de Medicina de la Universidad de Chile.

Estos dedos se amputaron con costotomo marca Meka $\odot$ en la articulación interfalángica entre falange proximal y media en dedos índices y en la articulación metacarpo-falángica en los pulgares.

La muestra consideró 9 individuos de sexo femenino y 7 individuos de sexo masculino. De los 32 dedos obtenidos, 16 fueron dedos pulgares; 9 femeninos y 7 masculinos, 7 derechos y 9 izquierdos. 16 fueron dedos índices; 9 femeninos y 7 masculinos, 7 derechos y 9 izquierdos. Todos estos dedos presentaban patrón dactiloscópico epidérmico externo visible y completo en la cara volar de la falange distal. Además todos ellos presentaban todas las capas de la piel y solo un dedo pulgar presentaba una cicatriz en su margen medial que no involucraba al núcleo papiloscópico.

Estos dedos no fueron fijados químicamente y solo se mantuvieron conservados bajo cadena de frío.

Los patrones dactiloscópicos fueron registrados con kit de toma de impresiones papilares marca Sirchie@ compuesto por: rodillo de caucho, tinta litográfica, reactivo negro de humo, planchuela, regla de apoyo, ficha dactiloscópica y lupa dactiloscópica. Además para la digitalización de estas impresiones, se utilizó scanner de imagen de color, marca HP@ y modelo Laserjet Pro400 m425dn, y se digitalizó a un mínimo de 300 Dpi. El procesamiento de imágenes se llevó a cabo con programa incluido con el Scanner. Para el registro fotográfico de todo el proceso se utilizó cámara fotográfica marca Nikon $@$, modelo D3200 con flash externo marca Nikon@ modelo speedlight SB-700.

Antes de su registro papiloscópico, se procedió a limpiar la epidermis con cepillo de cerdas suaves con una mezcla de agua con jabón neutro y fueron secados con gasa quirúrgica. Posteriormente se tomaron los registros físicos artificiales de patrones papiloscópicos epidérmicos y registros físicos artificiales de patrones papiloscópicos dérmicos.

Para estos registros del patrón dactiloscópico o papiloscópico, se utilizó la técnica de obtención de impresiones necrodactilares del Laboratorio de Identificación Forense perteneciente al Laboratorio de Criminalística de Carabineros de Chile (LABOCAR). 
En aquellos dedos que presentaron una deshidratación profunda, se utilizó inyección subdérmica de aproximadamente $3,5 \mathrm{ml}$ de suero fisiológico. Para desprender la epidermis de la dermis se utilizó inyección subdérmica de aproximadamente $3,5 \mathrm{ml}$ de hidróxido de amonio a una concentración del $100 \%$.

El cotejo dactilar entre los 2 registros físicos artificiales obtenidos de un dígito (relieve papilar epidérmico y el relieve papilar dérmico), se efectuó siguiendo el siguiente procedimiento de rutina denominado Cotejo Dactiloscópico, utilizado de forma universal en la identificación por técnica papiloscópica:

1.Se identifica y compara el diseño nuclear (verticilo, arco, presilla derecha y presilla izquierda).

2.Se cuentan las líneas que componen el diseño nuclear (desde el terminal interno al externo).

3. Se determina la ubicación de los puntos característicos con respecto a la región del dactilograma.

4. Se determina la situación del o los puntos característicos con respecto a la distancia de otros puntos característicos (se cuentan las líneas que los separan).

5. Se define su dirección, vertical, horizontal u oblicua.

6. Se busca una cantidad de puntos característicos coincidentes para afirmar identificación positiva.

Esta comparación o cotejo, fue realizada por peritos dactiloscópicos pertenecientes, formados, certificados y autorizados por el Laboratorio de Criminalística de Carabineros de Chile (LABOCAR).

\section{RESULTADOS}

Para una correcta comprensión se han dividido los resultados en epidérmicos, dérmicos y del cotejo identificatorio.

\section{Resultados de la observación de los registros físicos ar- tificiales de patrones papiloscópicos epidérmicos}

En la Tabla I, se puede observar la distribución de grupos o tipos fundamentales hallados en la cara volar epidérmica de dígitos de la muestra, es importante hacer notar que el tipo "Arco" no fue encontrado en la muestra. Por otro lado el tipo "Verticilo" es el más frecuente, llegando casi al $47 \%$ del total de la muestra. Por último, en uno de los dígitos (individuo de sexo masculino con rango de edad de 70 a 80 años) no fue posible determinar su grupo o tipo fundamental (debido a un desvanecimiento avanzado del dactilograma).

La Tabla II muestra la calidad y definición de los grupos o tipos fundamentales. Los resultados indican un alto porcentaje dígitos con TF borrosos $(37,5 \%)$. Siendo, este hecho mayor en hombres que en mujeres y mayor en individuos de edad avanzada (66,666\% sobre los 60 años).

La Tabla III indica la cantidad y calidad de puntos característicos. Estas estructuras morfológicas de la cara volar de los dígitos permiten el cotejo dactilar y la posterior identificación humana positiva.

Tabla I. Distribución de grupos o tipos fundamentales de la muestra en registro físico artificial de patrón papiloscópico epidérmico.

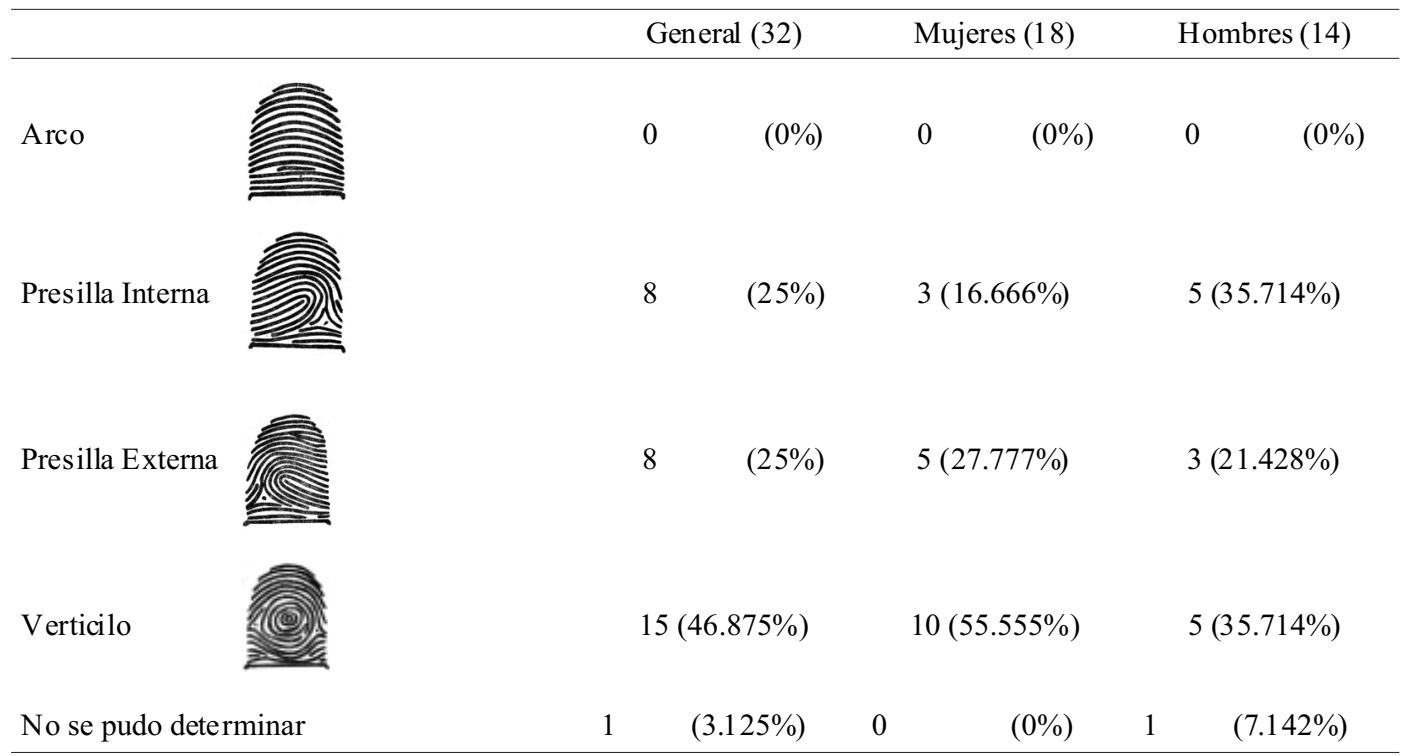


Tabla II. Calidad y definición de los grupos o tipos fundamentales en registro físico artificial de patrón papiloscópico epidérmico. Se consideró "buena" a aquella figura del registro físico artificial de patrón papiloscópico que pudiera ser identificada con facilidad. Se consideró "borroso" a aquel registro físico artificial de patrón papiloscópico que presenta un grado de desvanecimiento avanzado.

\begin{tabular}{lccc}
\hline & General (32) & Mujeres (18) & Hombres (14) \\
\hline Buena & $20(62,5 \%)$ & $14(77,777 \%)$ & $6(42.857 \%)$ \\
Borroso & $12(37,5 \%)$ & $4(22,222 \%)$ & $8(57.142 \%)$ \\
\hline
\end{tabular}

Tabla III. Cantidad y calidad de puntos característicos en registro físico artificial de patrón papiloscópico epidérmico. Se consideró "suficientes" cuando el número de PC nítidos y alojados en el núcleo papiloscópico sobrepasó los 9. Se “consideró ”insuficientes aquellos registros con PC menores a 9 o sin definición clara para ser reconocidos.

\begin{tabular}{lccc}
\hline & General (32) & Mujeres (18) & Hombres (14) \\
\hline Suficientes & $31(96,875 \%)$ & $18(100 \%)$ & $13(92,857 \%)$ \\
Insuficientes & $1(3,125 \%)$ & $0 \%$ & $1(7,142 \%)$ \\
\hline
\end{tabular}

Tabla IV. Presencia de cicatrices en registro físico artificial de patrón papiloscópico epidérmico y dérmico. Solo se encontró en un dígito pulgar perteneciente a individuo mujer en rango de edad de 60 a 70 años (tanto en su epidermis como en su dermis). Este dedo presento una cicatriz en la cara volar y en su zona marginal al núcleo papiloscópico, lo que no impide su cotejo.

\begin{tabular}{lccc}
\hline & General (32) & Mujeres (18) & Hombres (14) \\
\hline Presente & $1(3,125 \%)$ & $1(5,555 \%)$ & $0 \%$ \\
Ausente & $31(96,875 \%)$ & $17(94,444 \%)$ & $14(100 \%)$ \\
\hline
\end{tabular}

Tabla V. Presencia de líneas albas, blancas o seniles en la epidermis en registro físico artificial de patrón papiloscópico epidérmico.

\begin{tabular}{lccc}
\hline & General (32) & Mujeres (18) & Hombres (14) \\
\hline Presente & $21(65,625 \%)$ & $12(66,666 \%)$ & $9(64,285 \%)$ \\
Ausente & $11(34,375 \%)$ & $6(33,333 \%)$ & $5(35,714 \%)$ \\
\hline
\end{tabular}

La Tabla IV da cuenta de la presencia de cicatrices del total de la muestra. Se pudo encontrar 1 cicatriz en 1 pulgar de sexo femenino, ubicado en la región marginal (fuera de la región nuclear) de la cara volar del dígito en cuestión.

El hallazgo de líneas albas, blancas o senIles en la epidermis, se registró en la Tabla V. En esta muestra, se observaron 21 individuos que presentaron líneas seniles, 12 mujeres y 9 hombres $(65,625 \%$ del total de la muestra).

Finalmente, en epidermis (Tabla VI), se pudieron registrar 192 puntos característicos en los 32 dedos de la muestra. Los puntos característicos más frecuentes correspondieron a "extremo de línea" $(53,13 \%)$ y "bifurcaciónhorquilla” (41,15\%).

\section{Resultados de la observación de los registros físi- cos artificiales de patrones papiloscópicos dérmicos}

En la Tabla VII, se registró la distribución de grupos o tipos fundamentales observados en la cara volar dérmica de dígitos de la muestra, al igual que en epidermis, el tipo "Arco", no encontrado y por otro lado el tipo "Verticilo" fue el más frecuente, llegando casi al $47 \%$ del total de la muestra. A diferencia de los resultados epidérmicos, se constató que en un $12,5 \%$ de los casos no fue posible determinar el grupo o tipo fundamental (todos individuos de edad superior a 60 años).

La Tabla VIII nos muestra la calidad y definición de los grupos o tipos fundamentales. Los resultados indican un $75 \%$ de dígitos que presentaron TF borrosos y poco definidos $(66,666 \%$ de las mujeres, $85,714 \%$ de los hombres y un $83,333 \%$ de individuos de edad mayor a 60 años).

La Tabla IX indica la cantidad y calidad de puntos característicos, así en la dermis, fue mucho más dificultosa la observación de estas estructuras y solo el 40,625 \% de la muestra presenta una cantidad y calidad de puntos característicos que permiten el cotejo dactilar y la posterior identificación humana positiva.

Con respecto a las cicatrices encontradas en la muestra dérmica (Tabla IV), los hallazgos coinciden en número, posición y forma con lo observado en la muestra epidérmica.

La Tabla X resume las observaciones con respecto a la presencia de líneas albas, blancas o seniles. Se pudo constatar que solo un $12,9 \%$ de los individuos de la muestra fue posible observar la presencia de estas líneas características de individuos de edad avanzada.

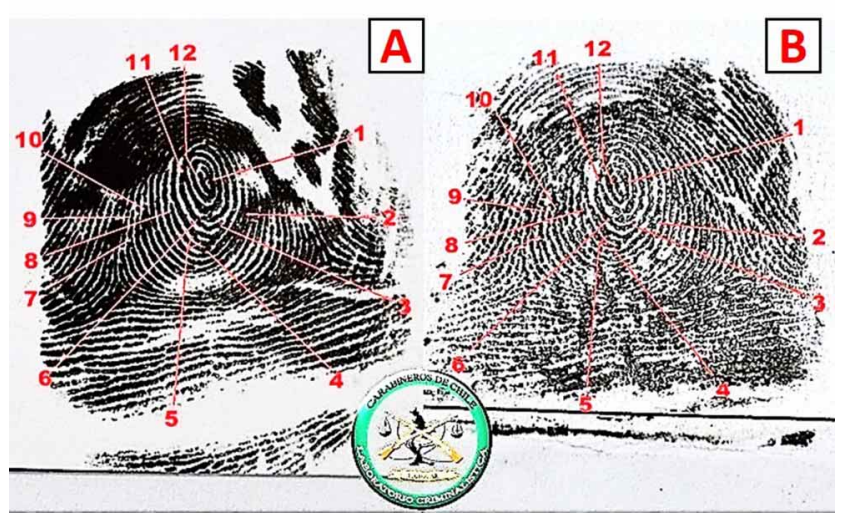

Fig. 3. Cotejo papiloscópico entre registro físico artificial de patrón papiloscópico epidérmico $(\mathrm{A})$ y registro físico artificial de patrón papiloscópico dérmico (B). Los números en rojo del 1 al 12, indican los PC semejantes entre ambos registros (tanto en forma como en posición), lo que permite la comparación positiva. 
Tabla VI. Puntos característicos observados en registro físico artificial de patrón papiloscópico epidérmico. Se consigna la frecuencia con la que se pudo observar cada PC en la muestra.

\begin{tabular}{|c|c|c|}
\hline Punto característico & Forma del PC & Presencia \\
\hline Total observado & & $192(100 \%)$ \\
\hline Extremo de línea & & $102(53,13 \%)$ \\
\hline Bifurcación y horquilla & & $79(41,15 \%)$ \\
\hline Encierro & & $6(3,13 \%)$ \\
\hline Gancho & & $2(1,04 \%)$ \\
\hline Islote & & $1(0,52 \%)$ \\
\hline Empalme & & $1(0,52 \%)$ \\
\hline Punto & & $1(0,52 \%)$ \\
\hline
\end{tabular}

Tabla VII. Distribución de grupos o tipos fundamentales de la muestra en registro físico artificial de patrón papiloscópico dérmico.

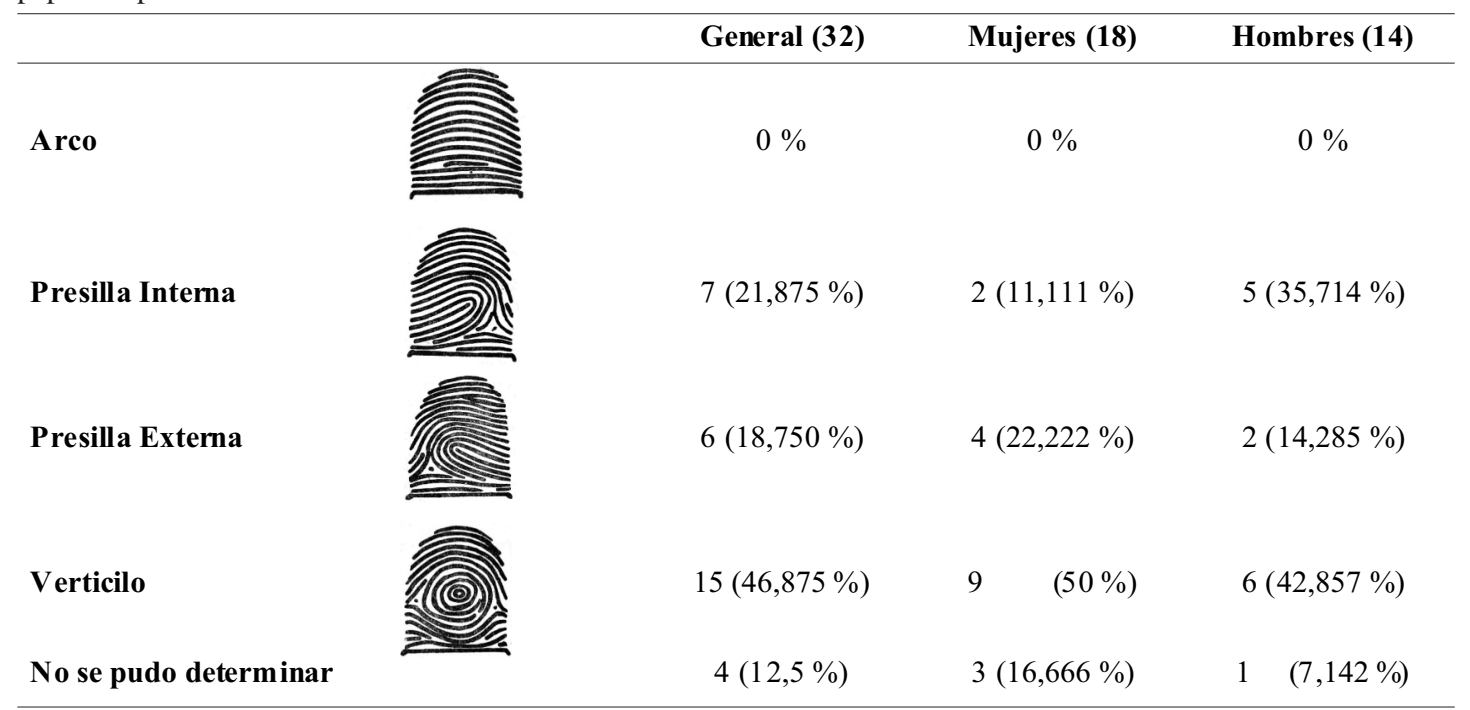


Tabla VIII. Calidad y definición de los grupos o tipos fundamentales en registro físico artificial de patrón papiloscópico dérmico. Se consideró "buena" a aquella figura del registro físico artificial de patrón papiloscópico que pudiera ser identificada con facilidad. Se consideró "borroso" a aquel registro físico artificial de patrón papiloscópico que presenta un grado de desvanecimiento avanzado.

\begin{tabular}{lcrr}
\hline & General (32) & \multicolumn{1}{c}{ Mujeres (18) } & Hombres (14) \\
\hline Buena & $8(25 \%)$ & $6(33,333 \%)$ & $2(14,285 \%)$ \\
Borroso & $24(75 \%)$ & $12(66,666 \%)$ & $12(85,714 \%)$ \\
\hline
\end{tabular}

Tabla IX. Cantidad y calidad de puntos característicos en registro físico artificial de patrón papiloscópico dérmico. Se consideró "suficientes" cuando el número de PC nítidos y alojados en el núcleo papiloscópico sobrepasó los 9. Se consideró "insuficientes" aquellos registros con PC menores a 9 o sin definición clara para ser reconocidos. Se consideró "no se observan" cuando fue imposible encontrar algún PC.

\begin{tabular}{lccc}
\hline & General (32) & Mujeres (18) & Hombres (14) \\
\hline juficientes & $13(40,625 \%)$ & $8(44,444 \%)$ & $5(35,714 \%)$ \\
nsuficientes & $12(37,500 \%)$ & $4(22,222 \%)$ & $8(57,142 \%)$ \\
Jo se observan & $7(21,875 \%)$ & $6(33,333 \%)$ & $1(7,142 \%)$ \\
\hline
\end{tabular}

Tabla X. Presencia de líneas albas, blancas o seniles en registro físico artificial de patrón papiloscópico dérmico.

\begin{tabular}{lccc}
\hline & General (32) & Mujeres (17) & Hombres (14) \\
\hline Presente & $4(12,903 \%)$ & $1(5,882 \%)$ & $3(21,428 \%)$ \\
Ausente & $27(87,096 \%$ & $16(94,117 \%$ & $11(78,571 \%)$ \\
\hline
\end{tabular}

Resultado del cotejo identificatorio: Al comparar los registros físicos artificiales papiloscópicos superficiales epidérmicos con los registros físicos artificiales papiloscópicos dérmicos, fue posible cotejar positivamente, a 13 individuos de la muestra (Fig. 3), en forma parcial a 8 individuos de la muestra y no se logró cotejar positivamente a 10 individuos de la muestra (Fig. 4).

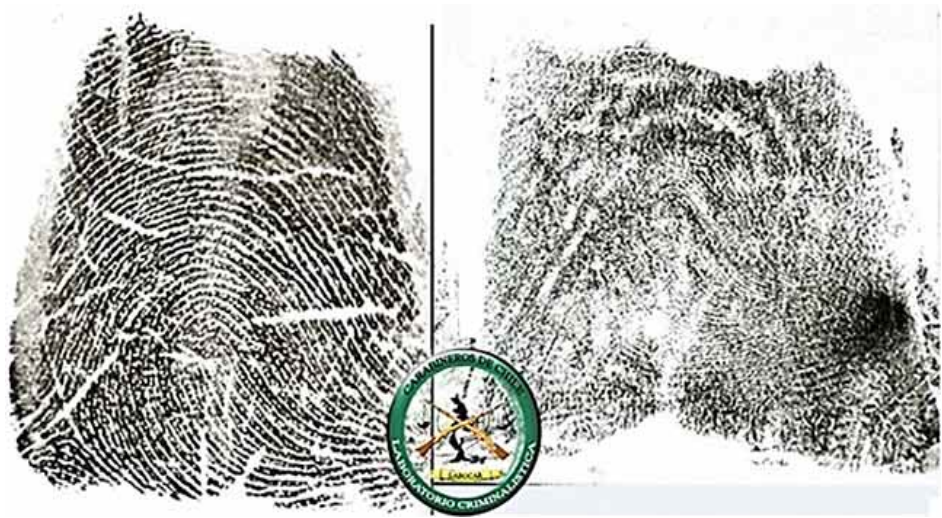

Fig. 4. Cotejo papiloscópico entre registro físico artificial de patrón papiloscópico epidérmico (A) y registro físico artificial de patrón papiloscópico dérmico (B). Debido a que el registro dérmico es de baja calidad, observándose borroso y poco nítido, no es posible realizar una comparación positiva.

\section{DISCUSIÓN}

Pese a la diferencia observada en los resultados de la distribución de TF entre la epidermis y la dermis (en epidermis se pudo reconocer TF en el $96,875 \%$ mientras que en dermis se pudo reconocer en el $87,5 \%$ ), no se encontró ningún dígito que presentara TF diferente entre su registro físico artificiales de patrón papiloscópico epidérmico y el registro físico artificial de patrón papiloscópico dérmico.

También se pudo constatar que los TF se observan borrosos y poco definidos mayoritariamente en los registros físicos artificiales de patrones papiloscópicos dérmicos (en registros de epidermis solo el 37,5 \% de la muestra presenta patrón papiloscópico borroso, mientras que registros de dermis se observó un $75 \%$ patrón papiloscópico borroso).

Otra diferencia notable entre los registros físicos artificiales de patrones papiloscópicos epidérmicos y dérmicos, es la cantidad y calidad de PC. Así, en registros de epidermis se pudo constatar un $96,875 \%$ de la muestra con PC suficientes mientras que en registros de dermis este valor solo llega al 40,625\% de la muestra. Además en este último grupo, existe un $21,875 \%$ de dígitos en que no es posible constatar PC.

También se pudo demostrar que existen algunas diferencias por sexo, tanto en la calidad como en la definición de TF y en la cantidad y calidad de PC.

Por último la diferencia encontrada en la presencia de líneas albas, blancas o seniles es importante, pudiendo observarse este rasgo morfológico con facilidad en epidermis, pero no es así en dermis $(65,625 \%$ de presencia en epidermis y un $12,903 \%$ en dermis). Este hecho pareciera indicar que la línea senil es un rasgo epidérmico y no tanto un rasgo epidérmico-dérmico.

Todos estos resultados son completamente nuevos y no se encuentran descritos en la literatura especializada.

Por otra parte, se observó gran cantidad de TF y PC epidérmicos borrosos y poco nítidos, hecho que concuerda con lo descrito por Silva et al. 
(2018), en referencia al desvanecimiento parcial o total que se ha observado y descrito en edades avanzadas.

Con respecto a la presencia de cicatrices tanto en epidermis como en dermis, los datos son concordantes con lo descrito en la literatura (Trujillo Arriaga, 2007).

Finalmente y con respecto al objetivo planteado en este trabajo, podemos afirmar que cuando los registros físicos artificiales de patrones papiloscópicos dérmicos son de calidad (TF y PC suficientes y nítidos) la relación morfológica certera con los registros físicos artificiales de patrones papiloscópicos epidérmicos está presente. Este hecho permite utilizar los registros físicos artificiales papiloscópicos dérmicos para la identificación humana positiva.

SILVA, J. ; ARAYA, C.; SOTO, M.; GONZÁlEZ, S.; SALCEDO, A.; BUSTOS, P.; ILUFI, I.; ORMAZÁBAL, J.; SANHUEZA, J.; PINO, A.; PLAZA, A. \& ARCOS, S. Relationship between the epidermal and the dermal dactyloscopic pattern. Int. J. Morphol., 36(4):1290-1297, 2018.

SUMMARY: The most used method in human identification is the dactyloscopy, which registers, analyzes and collates the fundamental types and characteristic points of figures present in the dactylogram determining the degree of coincidence, between a pattern of identity that is dubious, and one that is indubious. Due to the processes that affect the skin of corpses, such as putrefaction, the necropapiloscopy techniques that occupy the dermal patterns for human identification are used. The objective of the present work is to compare epidermal with dermal records and validate scientifically, this method of identification (epidermal - dermal). For this purpose, 32 cadaveric fingers of Chilean individuals, of both sexes and between 40 and 80 years were used. In order to obtain the epidermal and dermal artificial physical record, the technique of obtaining necrodactyle impressions was used and compared through of the technique of dactyloscopic comparison. These procedures were carried out by criminalistic experts of Carabineros de Chile. It was possible to show important differences between epidermis and dermis in terms of quantity and quality of characteristic points and presence of senile lines. In relation to fundamental types, it was evidenced that in the dermis it is difficult to observe fundamental types (blurred) but no digit was found that presented a different of fundamental type between epidermis and dermis. There were also differences by sex and age. Finally, it was possible to demonstrate that there is a similar morphological relationship between artificial physical records of epidermal papiloscopic patterns with dermal patterns from the same individual. This allows the use of dermal records for positive human identification. The results of this work are important in providing scientific evidence for human identification based on the dermal fingerprint pattern.

KEY WORDS: Necropapiloscopy; Dactylogram; Dactyloscopy; Papiloscopy; Human identification.

\section{REFERENCIAS BIBLIOGRÁFICAS}

Aliaga, D. Huellas Digitales. Santiago de Chile, Imprebess, 1988.

Beaven, C. Huellas Dactilares. Los orígenes de la Dactiloscopía y de la Ciencia de la Identificación Criminal. Barcelona, Alba, 2003.

Chacko, L. W. \& Vaidya, M. C. The dermal papillae and ridge patterns in human volar skin. Acta Anat. (Basel), 70(1):99-108, 1968.

de Antón y Barberá, F. Iniciación a la Dactiloscopía y Otras Técnicas Policiales. $3^{\mathrm{a}}$ ed. Valencia, Tirant Lo Blanch, 2005.

de Wilde, A. G.; van Meulenaarsgraf, H.; Plegging, J. H. \& AmeszVoorheove, H. Classifications of finger patterns. Coll. Anthropol., 5:13546, 1981.

Galton, F. Finger Prints. Londres, Mc Millan \& Co., 1892.

Gibbs, R. C. Fundamentals of dermatoglyphics. Arch. Dermatol., 96(6):721$5,1967$.

Girotti, S. Necropapiloscopía Papiloscopía Aplicada a la Identificación de Cadáveres. Buenos Aires, Dosyuna, 2014.

González Wood, A. Dactiloscopia Aplicada. Santiago de Chile, Tegualda, 1946.

Holder, E. H. Jr.; Robinson, L. O. \& Laub, J. H. The Fingerprint Sourcebook. Washington D. C., U. S. Department of Justice, Office of Justice Programs, 2012.

Holt, S. B. Quantitative genetics of finger-print patterns. Br. Med. Bull., 17:247-50, 1961

Ilabaca, F.; Palma, J. \& Henriquez, H. Métodos, Sistemas y Procedimientos de Identificación Humana. Santiago de Chile, Biblioteca del Detective, Ministerio de Defensa Nacional, Policía de Investigaciones de Chile, 1986.

Kücken, M. \& Newell, A. C. A model for fingerprint formation. Europhys. Lett., 68(1):141-6, 2004.

Kücken, M. \& Newell, A. C. Fingerprint formation. J. Theor. Biol., 235(1):71-83, 2005.

Kücken, M. Models for fingerprint pattern formation. Forensic Sci. Int., 171(2-3):85-96, 2007.

Lubian, R. Dactiloscopía. $4^{\mathrm{a}}$ ed. Madrid, Reus, 2006.

Reyna, L. Dactiloscopia Argentina, su Historia e Influencia en la Legislación. La Plata, Talleres Gráficos de Joaqui Sesé, 1909.

Rico Méndez, F. G. \& de Anda, D. La Fotografía Forense en la Peritación Legal. Ciudad de México, Trillas, 1991.

Silva, J.; Rojas, M.; Araya, C. \& Barraza, N. Pérdida del dactilograma durante la senilidad. Int. J. Morphol., 36(1):258-61, 2018.

Teke Schlicht, A. Medicina Legal \& Criminalística. Santiago de Chile, Metropolitana, 2010.

Trujillo Arriaga, S. T. El Estudio Científico de la Dactiloscopia. $2^{\mathrm{a}}$ ed. Ciudad de México, Limusa, 2007.

Vucetich, J. Dactiloscopía comparada. La Plata, Establecimiento tipográfico de Jacobo Pruser, 1904.

Dirección para correspondencia:

Prof. Dr. Juan Luis Silva R.

Académico - Investigador

Facultad de Medicina

Universidad San Sebastián

Campus Los Leones de Providencia

Dirección Lota 2465, Edificio F

Santiago - CHILE

Email: juan.silva@uss.cl

Recibido : 10-01-2018

Aceptado: 01-06-2018 